\title{
Quantitative Crystallographic and Statistical Image Processing for Materials Science in Scanning Transmission Electron Microscopy
}

\author{
Q.M. Ramasse ${ }^{1}$, M.C. Sarahan ${ }^{1,2}$, D.G. Morgan $^{3}$ and N.D. Browning ${ }^{4}$ \\ ${ }^{1}$ SuperSTEM Laboratory, STFC Daresbury Campus, Keckwick Lane, Daresbury WA4 4AD, U.K. \\ 2 Present address: Gatan Inc., 5794 W. Las Positas Blvd., Pleasanton CA 94588, U.S.A. \\ 3 Indiana University, Chemistry Department, 212 S. Hawthorne Dr., Bloomington, IN 47405, U.S.A. \\ ${ }^{4}$ Chemical and Materials Sciences Division, Pacific Northwest National Laboratory, 902 Battelle \\ Blvd., Richland, WA 99352 USA
}

An essential corollary to the exciting instrumentation developments electron microscopy has witnessed over the last two decades is the significant increase in detection limits and signal-to-noise $(\mathrm{S} / \mathrm{N})$ ratios achieved on the new generation of aberration-corrected microscopes, which provide greater flexibility and improved data collection ability. For scanning transmission electron microscopy (STEM) and analytical electron microscopy in particular, not only are large volumes of data being generated, many types of signal (images, spectra, diffraction patterns) are collected at the same time. This increases the need for advanced processing methods to make analyse and interpret such vast amounts of data. Multivariate statistical methods such as principal component analysis (PCA) or independent component analysis (ICA) are particularly powerful tools to mine large datasets for similarities or variations. These methods have for instance recently been employed to characterize dislocation cores in complex oxides, revealing pm-sized structural deformations and relating them to chemical variations: fig. $1[1,2]$. A statistical approach to processing is also essential when combining complementary signals (energy loss spectra, bright field and Z-contrast imaging...), in order to extract as much information as possible about the samples being investigated and yield a full structural and chemical picture of defects when associated with theoretical calculations [3].

Furthermore, with the developments of so-called 'gentle', dose-controlled, STEM techniques, materials scientists have turned their attention to many materials that were once extremely challenging to observe due to their sensitivity to radiation, including low-dimensional materials such as graphene. The materials science community is thus re-discovering and adapting low-dose image/data processing techniques that have long been employed in other fields, particularly those used in bio-EM. Analytical procedures such as crystallographic averaging can be particularly useful in obtaining precise structure information from noise-limited images and assess statistically the confidence with which this information can be interpreted. This can not only be applied in low dose imaging but also when attempting to push instrumental resolution to its limits in high dose images, for which the smallest spatial components are barely above noise levels. 2D crystallographic processing was thus instrumental in determining the guest site occupancy of silicon-based based clathrates developed for energy storage purposes [4]. Similarly, while averaging stacks of individual images with poor $\mathrm{S} / \mathrm{N}$ to build up a better picture of the observed structure has long been a staple of electron microscopy of organic materials, only recently have rigid registration techniques been widely employed to improve the precision in Z-contrast micrographs of inorganic sensitive materials: fig. 2 [5]. This means that precisions of a few pm are readily achieved in Z-contrast STEM images [6], allowing the precise direct experimental determination of the structure of defects for comparison with ab initio studies. 
References

[1] M.C. Sarahan et al., Ultramicroscopy 111 (2011), pp. 251-257

[2] H. Yang et al., Philosophical Magazine (2013), doi:10.1080/14786435.2012.746479.

[3] I. MacLaren et al., Advanced Functional Materials 23 (2013), pp. 683-689.

[4] D.G. Morgan, Q.M. Ramasse and N.D. Browning, J. Elec. Microsc. 53 (2009), pp. 223-244.

[5] J.P. Buban et al., Journal of Electron Microscopy 59 (2010), pp. 103-112.

[6] M. Saito et al., Journal of Electron Microscopy 58 (2009), pp. 131-136.

[7] A portion of this work was performed at PNNL which is operated by Battelle Memorial Institute for the US DOE under contract No. DEAC05-76RL01830. SuperSTEM is funded by the UK Engineering and Physical Sciences Research Council. Dr. Recep Zan is gratefully acknowledged for providing the $\mathrm{MoS}_{2}$ sample.
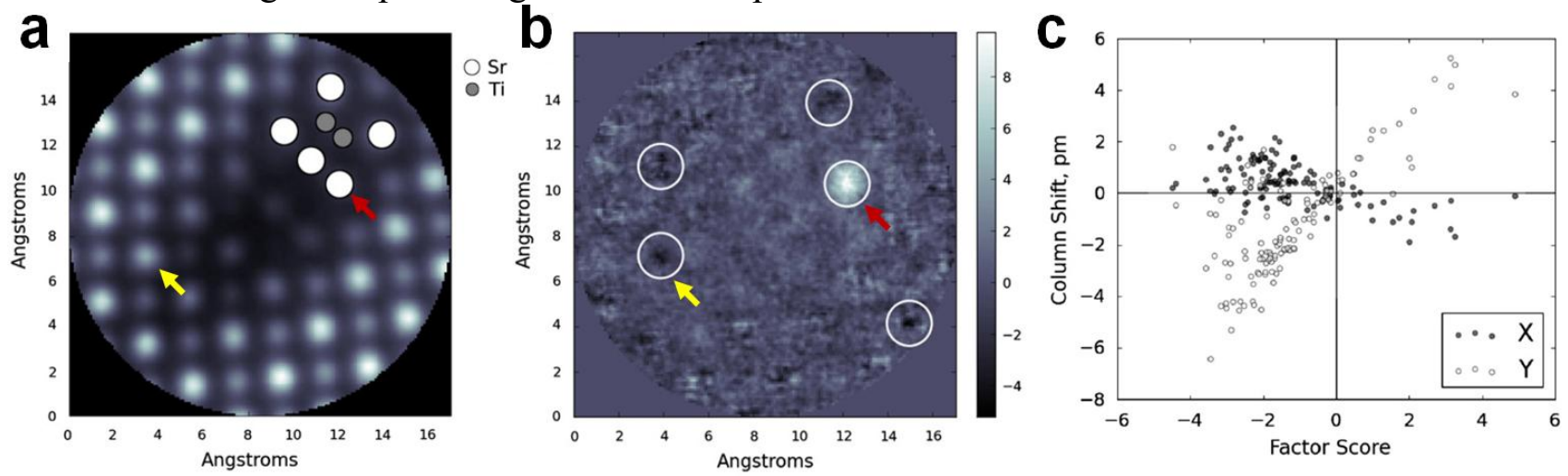

Figure 1. (a) Template-averaged Z-contrast image of a dislocation core in a $\Sigma 3$ grain boundary (GB) in $\mathrm{SrTiO}_{3}$. A postulated model of the core structure is overlaid. (b) So-called 'factor image' obtained through ICA analysis of many images of dislocation cores along the same GB, such as shown in (a). This reveals a trend throughout the datasets for a simultaneous increase in intensity at the position indicated by a red arrow and decrease at the other sites circled (darker contrast). (c) Careful interpretation of the ICA results shows that as the red arrow site intensity increases (higher occupancy), the site indicated by the yellow arrow shifts by fractions of a pm [1].
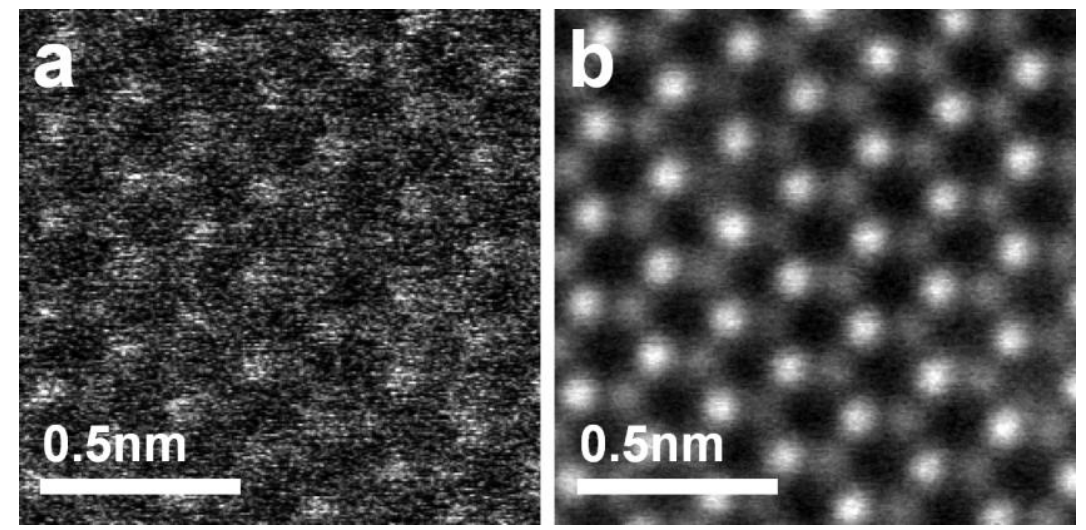

Figure 2. (a) Low S/N Z-contrast STEM image of a free-standing single layer sheet of topological insulator $\mathrm{MoS}_{2}$, a notoriously beam sensitive material. The image was acquired at $60 \mathrm{kV}$ acceleration voltage, with approximately $10 \mathrm{pA}$ of probe current and at a fast frame rate of $\sim 8$ frames $/ \mathrm{s}$. (b) Averaged image obtained by aligning and summing 100 consecutive frames, resulting in an estimated atomic position precision $<8 \mathrm{pm}$. 